\title{
«Of M.I.S. and men». Allocution du commissaire général Ferrier
}

Revue française d'automatique, d'informatique et de recherche opérationnelle. Recherche opérationnelle, tome 4, $\mathrm{n}^{\circ}$ V3 (1970), p. 5-9.

<http://www.numdam.org/item?id=RO_1970_4_3_5_0>

(C) AFCET, 1970, tous droits réservés.

L'accès aux archives de la revue « Revue française d'automatique, d'informatique et de recherche opérationnelle. Recherche opérationnelle » implique l'accord avec les conditions générales d'utilisation (http://www.numdam.org/ legal.php). Toute utilisation commerciale ou impression systématique est constitutive d'une infraction pénale. Toute copie ou impression de ce fichier doit contenir la présente mention de copyright.

\section{Numdam}

Article numérisé dans le cadre du programme

Numérisation de documents anciens mathématiques

http://www.numdam.org/ 


\section{OF M.I.S. AND MEN}

\section{ALLOCUTION DU COMMISSAIRE GENERAL FERRIER}

\section{NOTE DE LA REDACTION}

Nous sommes heureux de pouvoir publier quelques extraits du discours d'ouverture de la $3^{\mathrm{e}}$ Conférence organisée par l'OTAN sur les applications de la recherche opérationnelle aux grands problèmes logistiques (Luxembourg, mai 1970).

Le Commissaire Général de la Marine J. Ferrier est un pionnier dans ce domaine dont il a pu suivre l'évolution depuis l'époque des petits modèles jusqu'à l'âge des grands systèmes. Sous un titre malicieusement inspiré de Steinbeck, il propose quelques réflexions fort actuelles sur " les systèmes et les hommes " et nous met en garde contre un danger échappant aux concepts des mathématiciens : celui de suroptimiser.

La première conférence OTAN sur les systèmes d'approvisionnement eut lieu en septembre 1964. Elle avait pour titre : " les applications de la recherche opérationnelle aux problèmes militaires d'approvisionnement et de fourniture de services " ( $\left.{ }^{1}\right)$. L'exposé introductif en avait été confié à M. Murray A. Geisler, de la RAND. Cette estimable corporation n'avait guère plus de douze ans à l'époque. Aujourd'hui, bien qu'elle compte parmi les plus vénérables dames d'honneur de la Recherche Opérationnelle, elle est à peine sortie du " teen age ", de ces heureuses années d'apprentissage pendant lesquelles, si l'on n'atteint pas encore au sommet de la sagesse, on fait du moins provision d'expérience, en apprenant par

(1) Application of operational research methods to military servicing and supply problems. 
exemple, comme l'enseigne M. Durbin, à se méfier du "bottom up " $\left.{ }^{1}\right)$. M. Geisler nous fit quant à lui une belle démonstration dans le style " top down ". Du haut du Sinaï Californien où la RAND s'était déjà hissée en escaladant de 1957 à 1963 ses célèbres "laboratory problems ) LPI à LPIV, il sut donner au peuple élu de la logistique, que nous voulions être, un aperçu de la Terre Promise et l'espérance d'y pénétrer bientôt, à la remorque de ce que les Français appellent l'Informatique et qui, si l'on en croit la traduction récemment proposée par M. Kennedy au IVe Congrès de l'IFORS à Venise $\left({ }^{2}\right)$, n'est rien d'autre que l'application de la méthode scientifique à l'élaboration des systèmes d'information du management, désormais mondialement connus sous le sigle M.I.S.

La conférence de 1964 se pencha surtout sur des problèmes, je ne dirais pas mineurs, mais sectoriels. Il est normal que les petits garçons commencent par grimper aux arbres avant de dominer les forêts. Leurs naïves escalades peuvent d'ailleurs les amener assez haut et à voir assez loin. Ainsi je me rappelle avec enchante ment l'intervention d'un gentle man très britannique qui, fort de 30 années d'expérience vécue au contact d'avions cloués au sol par toutes les avaries possibles - Dieu sait si elles peuvent être nombreuses et variées - eut le talent de nous élever audessus des nuages tout en conservant les pieds sur la terre. Mr North sut nous faire sentir combien il est hasardeux d'obtenir, même en s'aidant de questionnaires savamment établis et imprimés, des réponses précises, c'est-à-dire des informations de base exactes, complètes, non subjectives. Or de telles informations sont la raison d'être des M.I.S. et le pain quotidien de tous nos systèmes logistiques. Il dépend des moissonneurs que les systèmes les plus subtils et les mieux agencés échappent ou non au désastreux bégaiement qui, en redoublant le M.I.S., transforme un management information system en ce que M. Russell Ackoff appelle un management misinformation system $\left({ }^{3}\right)$, avec toutes les suites hautement catastrophiques qui peuvent résulter d'un si déplorable avatar.

Trois ans plus tard, M. Sumner pinçait la même corde et lançait la conférence de 1967 dans le vent de l'Histoire en y donnant le signal de la première contestation.

Cette conférence avait pour titre et pour thème «les grands systèmes d'approvisionnement ». Il y avait progrès puisque l'on passait des problèmes aux systèmes et même aux grands systèmes, en somme de l'étude des mouvements à celle des machines. Progrès d'autant plus re marquable que le mot système, s'il n'était pas un nouveau venu, ne s'était pas encore

(1) Dans son exposé, intitulé information system design in large scale logistic systems, M. E. P. Durbin, de la RAND, établit un parallèle entre les deux modes de conception et d'évolution des systèmes informatiques, selon que l'élan créateur ou réformateur procède de la base (bottom up) ou du sommet (top down) de l'organisation.

(2) M. D. M. Kennedy, président de la session "informatics ", crut bon de définir dans son discours d'ouverture ce mot nouveau-né, traduit du Français " informatique » et récemment introduit dans la langue du management, version américaine. Il proposa : "informatics is the application of scientific methodology to the design of information systems for improved decision making ". La dernière précision paraît inutile, ou insuffisante, car le traitement de l'information débouche non pas seulement sur la décision, mais aussi bien sur la connaissance, le contrôle et le jugement.

(3) R. L. Ackoff (1967), Management Misinformation systems [ Mgmt/Sci. 14 (4)]. 
enrichi de tout ce que lui ont apporté les conquêtes récentes de la téléinformatique, les percées conceptuelles de la biocybernétique et les travaux de ceux que l'on commence à appeler, du moins en France, les " systématiciens".

Son contenu n'en était pas moins immense, déjà, dans le domaine qui nous intéresse. Ce fut l'occasion de l'inventorier. Nous êumes droit à la description, agréablement commentée, d'une bonne vingtaine de systèmes nés ou à naître, de tous types, et couvrant tous les horizons de la logistique depuis la prévision des besoins en pièces de rechange jusqu'à la cannibalisation des pièces en service et à l'évaluation des résultats finaux, avec en vedette Américaine, présentée par M. Craig C. Sherbrooke, le système M.E.T.R.I.C. dont ce fut la grande première $\left({ }^{1}\right)$.

Dans son discours d'ouverture, Robert G. Brown avait choisi de nous parler de l'architecture des systèmes et des tensions internes qui y prennent naissance $d u$ fait d'inévitables oppositions intellectuelles entre des administrateurs plus ou moins ineptes et des savants plus ou moins irresponsables. Il montra que pour surmonter ces oppositions, pour franchir ces interfaces, il n'est d'autre recours qu'en un dialogue véritablement ouvert et que les courbes d'échange sont le meilleur sinon le seul instrument d'un tel dialogue.

Ces idées ont trouvé en vous tous de profondes résonances. Vous êtes nombreux à souhaiter le renforcement et même l'élargissement d'un dialogue qui, du sommet à la base, devrait associer dans une compréhension commune de nos systèmes logistiques non seulement les hommes de science et les administrateurs mais aussi les constructeurs, les réparateurs et les distributeurs, les "mission planners ", les dirigeants et les exécutants, les officiers d'état-major et les combattants de première ligne voire, comme cela vient d'être splendidement démontré à Houston, les directeurs de vol et les naufragés de l'espace.

En 1967, notre deuxième conférence, placée sous le patronage de Minerve, fut gratifiée par Jupiter de deux coups de tonnerre.

Le premier fut frappé par M. Sumner. Il dénonça, avec toute l'autorité reconnue au représentant d'U.S. Eucom, la fragilité des systèmes monumentaux édifiés sur l'argile de la nature humaine et la vanité de vouloir faire toujours mieux en traitant toujours plus savamment des informations toujours plus raffinées, alors que les hommes chargés de récolter à la base des informations exactes et pertinentes, ce bien désormais souverain, risquent de comprendre de moins en moins ce qu'on attend d'eux et de s'en désintéresser de plus en plus.

La première partie de notre programme est plus spécifiquement consacrée aux thèmes directeurs qui font l'originalité de la prése nte

(1) Voir Large scale provisioning systems : J. Ferrier, éditeur, E.U.P. London et Dunod Paris : Les grands systèmes d'approvisionnement.

M.E.T.R.I.C. : Multi Echelon Technique for Recoverable Item Control. 
conférence. A côté de choses qui ont déjà été dites, mais qu'il est toujours bon de répéter, nous y avons rencontré des points de vue originaux, des idées neuves, des témoignages intéressants et des prises de position vigoureuses, comme celles tendant à élargir le dialogue et à resserrer l'interdépendance entre tous les partenaires du grand jeu de la logistique. On y trouve surtout un réel effort de surpassement afin de s'élever des modèles aux métamodèles, de l'analyse à la synthèse des systèmes, de la pratique à la théorie de la maintenance, des solutions de principe aux principes mêmes des solutions, des idées reçues à leur mise en cause, de la confrontation des points de vue à l'organisation du dialogue, du conformisme à la contestation puis à la participation. Il nous appartient à tous, compagnons d'une même cordée, de réussir cette belle escalade vers les temples sereins, chantés il y a 2000 ans par Lucrèce, d'où les sages peuvent contempler avec sérénité le tumulte parfaitement organisé des opérations savamment soutenues, qu'elles soient civiles, militaires ou, pour reprendre l'image du poète, maritimes : Suave mari magno, turbantibus aequora pentis...

Craignons pourtant de nous élever si haut et si droit, dans les sphères silencieuses des systèmes, que nous perdions de vue les pauvres exécutants, demeurés prisonniers de l'espace tangentiel du Commandant Sallantin ( ${ }^{1}$ ), du plan trivial de M. Koestler $\left({ }^{2}\right)$, de la terre des hommes de Saint Exupéry de ce monde rampant, plein de bruit et de fureur, ô Shakespeare, mais le seul en vérité où il se passe des choses réelles. Craignons de ne plus entendre le cri d'alarme lancé il y a trois ans à Athènes et, à force de marcher le nez tendu vers les étoiles de l'optimisation, craignons de nous retrouver, comme l'astronome de la fable, emprisonnés au fond d'un puits, même si c'est un puits de science.

Permettez-moi de conclure sur un souvenir personnel datant de ma toute première campagne. Cela se passait à l'Ecole Polytechnique, à l'époque déjà bien lointaine où les "jeunes savants ", comme on nous appelait alors avec une affectueuse ironie, étaient unanimement fiers d'être des militaires, de porter un uniforme, d'obéir à une discipline et d'avoir comme devise : "pour la patrie, les sciences et la gloire ". On nous faisait payer ces divers avantages en nous apprenant le maniement des armes, en particulier celui de l'épée. Notre instructeur, l'adjudant-chef Eckmann, avait fait ses classes et même la grande guerre (celle de 19141918) du côté Allemand. Cette singularité qui lui valait toute notre respectueuse considération se traduisait par un extrême souci de la perfection. J'éprouvais, comme beaucoup de jeunes mathématiciens, une certaine difficulté à tenir mon épée bien droite au moment du "Présentez Armes! " et j'entends encore la voix câline de l'adjudant Eckmann

(1) Les études épistémologiques du capitaine de frégate Sallantin sur l'algèbre naturelle, clef " informatique " et règle $d u$ jeu de toutes nos connaissances, mettent en relief la nature dialectique de quatre "dimensions " fondamentales parmi lesquelles figure l'espace, à la fois " tangentiel "et " vertical ". Ces travaux encore trop peu connus font l'objet de fascicules édités par le C.I.R.O. (centre interarmées de recherche opérationnelle).

(2) Voir le Yogi et le Commissaire : les mémoires d'Hillary. 
m'exhortant à la redresser : " La la me verticale, M. Ferrier,. bien verticale, encore un peu plus verticale! Mais non! N'exagérez pas!".

Messieurs ! Élevons nos lames, nos esprits, nos observatoires et les piliers de nos temples à la verticale. Mais sans exagération! Sachons nous ménager une perspective un peu rasante, pour ne point perdre le sens du relief, done de la réalité des choses, de leur éloignement et de leur pesanteur. C'est indispensable, même quand on va visiter la Lune. Lorsque nous allâmes pour la première fois visiter Minerve, à Athènes, en 1964, le grand péché contre l'esprit de la recherche opérationnelle, celui contre lequel fulminaient toutes les têtes bien remplies d'algorithmes, était de sous-optimiser. La crainte d'y succomber nous a conduits vers les systèmes très fortement intégrés qui eurent les honneurs de la deuxième conférence. Au moment d'aller encore plus haut et plus loin dans la voie de la perfection, prenons conscience qu'un autre danger nous menace, qu'un autre péché nous guette : celui de suroptimiser. Nous nous y exposons chaque fois que nous prenons le risque de fonder notre conception des M.I.S. sur une fausse conception, une "misconception" des hommes. Afin de mieux y échapper, ne manquons jamais d'introduire dans nos cocktails cybernétiques à base d'esprit de géométrie et d'esprit de système, un soupçon d'esprit de charité et de finesse, une larme de sweetspare, l'angustura du management. 